\title{
LA SUPERFICIALIDAD EN LA
} ENSEÑANZA DEL CONCEPTO DE ENERGÍA: UNA CAUSA DEL LIMITADO APRENDIZAJE ALCANZADO POR LOS
ESTUDIANTES DE BACHILLERATO

\section{THE SUPERFICIAL TEACHING OF THE ENERGY CONCEPT: A CAUSE OF THE LACK OF LEARNING ACHIEVED BY STUDENTS WHEN THEY FINISH THEIR STUDIES IN HIGH SCHOOL}

Josep Lluís Doménech, Rubén Limiñana, Asunción Menargues

Departamento de Didáctica General y Didácticas Específicas

Universidad de Alicante

jl.domenech@ua.es, ruben.lm@gmail.com, a.menargues@ua.es

RESUMEN: Hay un amplio acuerdo entre los investigadores en que una enseñanza en profundidad de los conceptos y modelos favorecerá su aprendizaje. El análisis de las propuestas y afirmaciones hechas por profesores en formación y en activo, en relación con la enseñanza de la energía en bachillerato (17/18 años), nos permite afirmar que la enseñanza habitual deja de lado aspectos importantes de dicho concepto. Esta sería una de las razones que explicaría la escasa comprensión con que terminan los estudios los alumnos de secundaria.

PALABRAS CLAVE: energía, enseñanza habitual, aprendizaje, epistemología.

ABSTRACT: There is a ubiquitous agreement amongst researchers that an in-depth teaching of concepts and models will promote learning. The analysis of the proposals and statements made by teachers about the energy teaching in high school (17/18 years-old students) allows stating that the usual education does not take into account relevant issues of that concept. This would be one of the reasons which explains the lack of the understanding of several concepts acquired by students they finish their studies in high school.

KEY WORDS: energy, usual education, learning, epistemology. 


\section{PLANTEAMIENTO DEL PROBLEMA E HIPÓTESIS}

La enseñanza de la energía constituye uno de los problemas recurrentes en la investigación en didáctica de las ciencias (Doménech, 2000). En todos los casos la conclusión es la misma: una mayoría de alumnos terminan los estudios de secundaria sin una comprensión adecuada de dicho concepto.

Algunas investigaciones han ido más allá de esta constatación y han mostrado las mejoras en el aprendizaje conseguidas con propuestas de enseñanza distintas de la habitual (Doménech, 2000; Solbes y Tarín, 2004; Varela y Pérez, 2006). Otras (Solbes y Tarín, 1998; Doménech et al., 2001...) han fijado la atención en la enseñanza habitual con el objetivo de encontrar razones que expliquen las deficiencias del aprendizaje alcanzado. El presente trabajo se inserta en esta segunda línea de investigación.

Frente a los cursos panorámicos, en los que se estudia un poco de todo y en donde necesariamente prima la superficialidad, existe un consenso entre los investigadores en anteponer la profundidad a la amplitud a la hora de elegir los contenidos que se deben tratar (Linn, 1986; Gil et al., 1992; Duschl, 1997; Vosniadou et al., 2001). La complejidad que supone la comprensión de los conocimientos científicos aconseja un estudio detenido de los conceptos y modelos, de manera que los estudiantes perciban la relevancia y el interés de los problemas que se van a tratar, el significado de los conceptos introducidos, su capacidad para resolver los problemas, las implicaciones sociales y tecnológicas de dichos conocimientos, etc., como requisito para un buen aprendizaje.

Con este punto de partida, y limitándonos al campo estrictamente conceptual, nos hemos planteado el siguiente problema: ¿contemplan los profesores los diferentes aspectos que permiten una comprensión adecuada del concepto de energía en bachillerato? Nuestra hipótesis es que los profesores dejan de lado aspectos fundamentales con relación a dicho concepto, lo cual constituye una de las causas de la deficiente comprensión que los alumnos de la secundaria superior alcanzan de la energía.

\section{FUNDAMENTACIÓN DE LA HIPÓTESIS}

Nuestra suposición se basa tanto en que la enseñanza habitual no dedica una atención adecuada a cómo se construyen los conocimientos científicos como en las deficiencias metodológicas de dicha enseñanza.

Investigadores y profesores coinciden en que el aprendizaje de los conceptos se facilitará en la medida en que los alumnos perciban cómo se trabaja en ciencias (Duschl, 1997). Una estrategia pertinente al respecto consiste en que los estudiantes trabajen en las clases de una manera parecida a como lo hacen los científicos. A la luz de lo que hoy en día se admite como característico del trabajo científico, nos encontramos con que la introducción de conceptos o modelos en la enseñanza debería tener como propósito resolver problemas (Chalmers, 1984 y 1992; Brown, 1988). Los conceptos y modelos se introducirán a título de hipótesis y habrá que poner a prueba su validez de diferentes maneras (diseñando y realizando experiencias de laboratorio, constatando la validez de las predicciones cualitativas y cuantitativas, etc.). En este proceso surgirán relaciones entre los nuevos conceptos y otros que se habían introducido previamente, y que estaban ya disponibles, y deberá constatarse la coherencia entre sus significados e interpretaciones.

Un enseñanza de la energía que quiera parecerse a la manera de trabajar en ciencias exige, entre otras cosas, enfrentar a los alumnos a los problemas que se pretenden resolver con su introducción, construir alguna concepción cualitativa al respecto y evidenciar su evolución a medida que se usa para resolver los problemas planteados, mostrar el campo de validez de dicha concepción, su conexión con conceptos relacionados (trabajo y calor), proponer expresiones para su medida y utilizarlas para hacer predicciones cuantitativas, etc. En definitiva, una enseñanza que quiera subrayar cómo se construyen los conocimientos científicos demanda un estudio detenido de todo lo relacionado con la energía. El 
hecho de que la enseñanza habitual deje de lado aspectos importantes del trabajo científico (McComas, 1998; Fernández, 2000) nos hace suponer que los profesores no considerarán aspectos decisivos de lo que supone una comprensión pertinente de la energía.

Por otro lado, según los modelos constructivistas del aprendizaje, las personas elaboran significados a partir de lo que ven u oyen, generando relaciones entre sus conocimientos previos y los nuevos fenómenos (Osborne y Wittrock, 1983 y 1985; Driver, 1988; Novak, 1998). El aprendizaje no supone simplemente la adición de nuevos conocimientos: los nuevos conocimientos afectan al significado de los ya existentes (Welti, 2005). Esta interpretación de lo «nuevo» en términos de lo «viejo» refleja una concepción del conocimiento, no como una serie de hechos aislados, sino, más bien, como altamente interrelacionados (Osborne y Wittrock, 1985; Campelo, 2003; de Oliveira et al., 2006).

Desde las concepciones constructivistas, el aprendizaje se verá favorecido en la medida en que los estudiantes dispongan de oportunidades para relacionar los conocimientos que han de aprender con sus estructuras conceptuales previas, y también para explorar sus implicaciones, hacer predicciones y conectarlos con otros conceptos. Es así que también desde el campo de la psicología del aprendizaje se recomienda un tratamiento en profundidad, un estudio detenido, de la energía. El que la enseñanza habitual esté bastante alejada de aquello que caracteriza a las estrategias constructivistas (Calatayud et al., 1992; Roth, 1994; Tobin et al., 1994; Porlán, 1995; Newton et al., 1999) nos lleva a suponer que la energía no se estudia con la atención requerida.

\section{METODOLOGÍA}

Muestra. Para poner a prueba la hipótesis sugerida hemos trabajado con tres grupos de profesores. Un grupo estaba constituido por 31 profesores en formación que en el momento del estudio cursaban el CAP (curso de capacitación para poder impartir docencia en secundaria) de la materia de Física y Química.

El resto eran profesores en activo de secundaria de Física y Química. Uno de los grupos estaba formado por 28 profesores, con una variada experiencia docente, que asistían, en el momento del estudio, a un curso de formación del profesorado sobre el problema de la enseñanza y el aprendizaje de la energía en bachillerato.

El tercer grupo estaba formado por 5 profesores. Los criterios para su elección fueron la experiencia docente (más de 5 años impartiendo enseñanza) y la proximidad de su centro de trabajo al lugar de residencia de uno de los autores de la investigación.

La razón de trabajar por separado con profesores en formación y en activo es que se trata de colectivos con un grado de formación y experiencias diferentes. Mientras los profesores en activo llevaban años ejerciendo la docencia, los profesores en formación no tenían ninguna experiencia docente. En principio, por tanto, deberían esperarse resultados distintos.

Instrumentos y métodos. Con el objetivo de averiguar los aspectos que consideran importantes con relación a la comprensión conceptual de la energía se les planteó, por escrito, una cuestión abierta. La cuestión se propuso como actividad inicial tanto a los profesores en formación como al grupo de profesores en activo. La actividad se planteó en nuestra presencia y los profesores dispusieron de tiempo ilimitado para su respuesta.

Los investigadores valoraron por separado las propuestas hechas. Tras el análisis de las respuestas de diez profesores, se hizo una puesta en común con la finalidad de aumentar la coherencia del análisis. El grado de coincidencia fue prácticamente total (superior al 95\%) y en las discrepancias se escogió la opción más desfavorable para nuestra hipótesis.

A fin de averiguar cómo introducen en las clases todo aquello relacionado con la energía (si dedican el tiempo suficiente a su estudio, su valoración sobre el aprendizaje alcanzado por los estudiantes, etc.), se decidió entrevistar a cinco profesores suficientemente experimentados. 
Cuestionario. Elaboramos una cuestión de prueba que trasladamos a tres profesores expertos para que valorasen su pertinencia y sugiriesen modificaciones. Después de este proceso, la cuestión quedó redactada de la siguiente forma:

1. Imagina que has de explicar los temas relacionados con la energía a estudiantes de bachillerato (17/18 años). Expresa qué tratarías de conseguir, es decir, qué crees que deberían llegar a saber los estudiantes de este campo de conocimientos. (Intenta precisar: no se trata de escribir, por ejemplo, «que comprendan qué es la energía», sino de indicar qué deberían llegar a comprender sobre la energía). Señala también las dificultades a las que deberíamos prestar atención.

Red de análisis. El análisis de las propuestas hechas por los profesores exige clarificar los aspectos que con relación a la energía deberían abordarse en las aulas de bachillerato.

En otro estudio (Doménech et al., 2007), hemos desarrollado el conjunto de aspectos relacionados con la energía que deben tenerse en consideración en una enseñanza que pretenda ir más allá de planteamientos puntuales. Algunos de ellos están relacionados con la necesidad de motivar a los alumnos, de hacerlos partícipes del interés y la importancia de esta problemática, otros tienen que ver con planteamientos didácticos, etc. La extensión que supone el análisis de un número tan elevado de aspectos nos obliga a limitar nuestro campo de estudio. Puesto que estamos interesados en los aspectos conceptuales, nos centraremos en esta parcela.

Limitándonos a lo puramente conceptual, podemos decir que un tratamiento en profundidad de la energía en bachillerato exige:

1. En relación con los problemas que condujeron a la introducción del concepto de energía, y de todo el cuerpo de conocimientos asociado, hemos de referirnos a los cambios, las transformaciones de la materia, buscando conexiones entre ellos.

2. Podemos asociar la energía, en una primera aproximación, a la capacidad de los sistemas para producir transformaciones (Chisholm, 1992; Arons 1997). La energía no es una especie de fluido, ni constituye el «combustible» necesario para producir cambios, como a menudo conciben los estudiantes (Ogborn, 1986; Trumper y Gorsky, 1993), sino que se trata de una propiedad que viene determinada por la configuración del sistema. Además, el término energía está referido siempre a un sistema físico en particular (que conviene explicitar).

3. Cuando hablamos de la energía potencial gravitatoria de una piedra, sabemos que ella es debida a la interacción entre la piedra y la Tierra y, por tanto, pertenece al conjunto formado por las dos y no solo a la primera (Mallindkrodt y Leff, 1992).

4. En contra de lo que se suele creer, no es posible determinar el valor absoluto de la energía de un sistema: solo podemos determinar sus variaciones (Beynon, 1990; Chisholm, 1992; Prideaux, 1995).

5. Cualitativamente podemos concebir el trabajo como la manera de cambiar la energía de un sistema debido a fuerzas que se desplazan.

6. El calor supone la manera de cambiar la energía de un sistema por medio de diferencias de temperatura. La energía interna es un concepto distinto del calor y está relacionada con la energía de las partículas constituyentes de la materia: los cuerpos no poseen calor, sino energía interna.

7. Las variaciones de energía de un sistema, $\Delta \mathrm{E}$, pueden ser debidas a la realización de trabajo, $\mathrm{W}$, y/o calor, $\mathrm{Q}$, de acuerdo con la expresión $\mathrm{W}+\mathrm{Q}=\Delta \mathrm{E}$. ${ }^{1}$

8. Se ha de destacar el avance que supuso la integración de la mecánica y el calor, que permitió el establecimiento del principio de conservación de la energía (Kuhn, 1983; Harman, 1990).

1. Dejamos para otro nivel el estudio de la radiación. 
9. Los cambios experimentados por los sistemas comportan transformaciones de unas formas de energía en otras y/o «transferencias» de energía de unos sistemas a otros (o de unas partes del sistema a otras), pero la energía de un sistema aislado permanece constante. Hemos de advertir, en contra de una derivación de dicho principio a partir del teorema de las fuerzas vivas, que «La ley de la conservación de la energía no es derivable de las leyes de la dinámica del movimiento; es una afirmación independiente sobre el orden en la naturaleza» (Arons, 1989).

10. Como resultado de las interacciones y consiguientes transformaciones de los sistemas, la energía se dispersa, se degrada. La dispersión de la energía disminuye la posibilidad de futuras transformaciones macroscópicas. Cuando hablamos de "consumo de energía», "crisis energética», etc., no queremos decir que la energía desaparezca, sino que se dispersa y deja de ser útil (la configuración del sistema ya no permite que continúen ocurriendo cambios macroscópicos) (Duit, 1986; Ogborn, 1986). El hecho de que la energía se conserve, pero no así la capacidad para producir transformaciones, afecta a la concepción cualitativa de energía propuesta, y los estudiantes han de ser conscientes de ello, pero dejamos para cursos posteriores la elaboración de una concepción más adecuada para la energía.

A la luz de esto hemos elaborado la siguiente red de análisis:

a) La energía es un concepto especialmente útil cuando nos enfrentamos de una manera general al estudio de las transformaciones que ocurren a nuestro alrededor. En concreto, las transformaciones que experimentan los sistemas están asociadas a variaciones de energía.

b) Es necesario explicitar el sistema físico objeto de estudio cuando se utiliza dicho concepto.

c) Solamente podemos determinar las variaciones de energía experimentadas por los sistemas, no sus valores absolutos.

d) Trabajo y calor son dos maneras diferentes de modificar la energía de un sistema.

e) La energía interna es un concepto diferente del calor.

f) Para el caso de un sistema aislado, la energía se mantiene constante (aun cuando ello no significa que el sistema no pueda experimentar cambios).

g) Como consecuencia de las interacciones con otros sistemas, la energía de un sistema se «degrada».

h) Se toman en consideración las posibles ideas alternativas de los estudiantes al respecto.

Entrevistas. Previamente a las entrevistas, y teniendo en cuenta la red de análisis, diseñamos una secuencia de interrogantes a plantear y la pasamos a dos investigadores expertos para que la considerasen y sugiriesen las modificaciones oportunas. A partir de ello, elaboramos la secuencia que se presenta en el apéndice, y con la cual entrevistamos a cinco profesores.

Las entrevistas se grabaron y se transcribieron. A fin de no fatigar a los entrevistados se decidió que su duración no excediese los 45 minutos. Los entrevistados dispusieron de papel y lápiz para ayudarse en sus explicaciones. Al finalizar, todos los profesores indicaron haberse sentido a gusto durante la entrevista y que sus afirmaciones reflejaban sus ideas sobre la enseñanza y el aprendizaje de la energía.

\section{ANÁLISIS DE LOS RESULTADOS Y DISCUSIÓN}

En la tabla 1 se presentan las propuestas hechas por los profesores cuando les pedimos que señalaran qué deberían conocer los estudiantes acerca de la energía. 
Tabla 1.

Aspectos apuntados por los profesores con relación a la energía (\%)

\begin{tabular}{|l|c|c|c|c|}
\hline & \multicolumn{2}{|c|}{ Formación } & \multicolumn{2}{c|}{ Activo } \\
\cline { 2 - 5 } & $\mathrm{N}=31$ & $\mathrm{~s}_{\mathrm{p}}$ & $\mathrm{N}=28$ & $\mathrm{~s}_{\mathrm{p}}$ \\
\hline Se refieren a las transformaciones que experimentan los sistemas & 23 & 0,08 & 32 & 0,09 \\
\hline El término energía está ligado al sistema físico objeto de estudio & 10 & 0,05 & 11 & 0,06 \\
\hline Solo podemos determinar variaciones de energía, no valores absolutos & 13 & 0,06 & 18 & 0,07 \\
\hline Trabajo y calor son dos maneras diferentes de cambiar la energía & 23 & 0,08 & 36 & 0,09 \\
\hline Hacen referencia al principio de conservación de la energía & 55 & 0,09 & 43 & 0,09 \\
\hline La energía interna es un concepto diferente del calor & 16 & 0,07 & 18 & 0,07 \\
\hline Mencionan la degradación de la energía & 6 & 0,04 & 32 & 0,09 \\
\hline Se refieren a posibles ideas alternativas de los estudiantes & 16 & 0,07 & 36 & 0,09 \\
\hline
\end{tabular}

Constatamos que son pocos los aspectos apuntados. En el caso de los profesores en formación, la conservación de la energía es el más señalado (55\%). El resto de aspectos son sugeridos por la tercera parte o menos. Indicaremos que estas propuestas coinciden básicamente con las hechas por estudiantes de bachillerato (Doménech, 2000) o de los primeros cursos de licenciaturas de ciencias (Pintó, 1991). Sorprende que solo el 6\%, 2 de los 31 profesores a quienes se les ha pedido opinión, mencionen la degradación como un aspecto que se deba considerar con relación a la energía. Esto significa que una gran mayoría ignoran la contribución del segundo principio de la termodinámica a la concepción científica de energía. El escaso número de profesores que se refieren a las ideas alternativas de los estudiantes sobre la energía evidencia que una gran parte desconocen la existencia de concepciones alternativas en los alumnos, ideas que dificultan el aprendizaje de su significado científico.

Como era de esperar, los profesores en activo apuntan más aspectos. No obstante, constatamos que el más citado (de nuevo, el principio de conservación de la energía) es sugerido por menos de la mitad.

El hecho de que únicamente la quinta parte de los profesores en activo distingan entre energía interna y calor podría explicar las confusiones al respecto mantenidas por los alumnos (Sevilla, 1986; Mak y Young, 1987). Así mismo, el que solo la tercera parte vean en el trabajo y en el calor maneras de cambiar la energía es coherente con el escaso porcentaje de estudiantes que perciben dicha conexión (Doménech y Martínez Torregrosa, 2010).

A continuación, presentamos algunos de los resultados obtenidos en las entrevistas.

1. Empezamos por averiguar cómo inician el tema, es decir, cómo justifican a sus alumnos el estudio que van a comenzar.

Ninguno de los profesores menciona que se va a proceder al estudio de las transformaciones que ocurren en la naturaleza. Dos profesores señalan que el propósito del tema consiste en estudiar los movimientos de una manera distinta a como se ha hecho en cinemática y dinámica, pero sin indicar qué se persigue con dicho estudio, qué lo diferencia:

Entrevistador Sabemos que, por ejemplo, los conceptos de velocidad y de aceleración se introducen en clase con el propósito de describir los movimientos; y que el concepto de fuerza se introduce con el objetivo de explicar los cambios de movimiento experimentados por los objetos, pero, ¿qué pretendemos explicar con el concepto de energía?, ¿qué les dices a los alumnos?

Profesor 1 Siempre la introducimos ligada al trabajo, nunca por separado.

Entrevistador ¿ ¿Y cómo empiezas? 


\section{Profesor 1 Primero trabajo.}

Entrevistador ¿ ¿Y cómo introduces el trabajo? Es decir, ¿cómo empiezas el tema?

Profesor 1 Lo ligo a la cinemática y a la dinámica y les digo que cuando las fuerzas producen un desplazamiento están produciendo un trabajo. A partir de esto les planteo diferentes casos, las diversas posibilidades, que el trabajo no es vectorial, sino que es un valor.

Les digo que vamos a estudiar el movimiento con otras magnitudes. Magnitudes escalares en vez de vectoriales. Les digo que verán que muchos problemas, que en mecánica eran difíciles de resolver, de esta manera son más fáciles. (Profesor 2)

Los otros tres profesores ni tan siquiera se refieren a los movimientos y empiezan directamente introduciendo conceptos:

Profesor 5 Es que el concepto de energía, la verdad es que es difícil. Yo empiezo con el tema de trabajo: una fuerza produce desplazamiento y luego lo relacionas con la energía cinética y con la energía potencial.

Entrevistador Pero, ¿qué justificación das a los alumnos para hacer esto?

Profesor 5 No sé... normalmente siempre hemos empezado con el concepto de trabajo.

Entrevistador ¿Así, sin más?

Profesor 5 Hombre, acabas de estudiar las fuerzas, y sabes que la fuerza produce un desplazamiento y podías... si una fuerza produce un desplazamiento... y te metes con el trabajo. Y luego relacionas el trabajo con la variación de energía cinética y con la variación de energía potencial. Esto es lo que normalmente se ha hecho.

2. La introducción de ideas cualitativas no solo ayuda a enfrentarse a los problemas y situaciones que se plantean con argumentos, sin fórmulas, sino que además sirve de guía para proponer las definiciones operativas. Por ello les hemos preguntado si introducen alguna concepción cualitativa de trabajo (el primer concepto que, según manifiestan, introducen) y de energía.

Ningún profesor propone una concepción cualitativa de trabajo. En todos los casos, la definición operativa $W=|\vec{F} \| \Delta \vec{r}| \cos \alpha$ se introduce sin aportar razón alguna:

Entrevistador ¿Cómo introduces el concepto de trabajo?

Profesor $2 \quad \mathrm{Al}$ principio, sin ninguna justificación.

Se trata de un planteamiento marcadamente operativista que sin dudar introduce una cierta arbitrariedad en la ciencia: ¿por qué definimos el trabajo a partir de la expresión $W=|\vec{F} \| \Delta \vec{r}| \cos \alpha$ y no $W=|\vec{F}| \Delta \vec{r} \mid \operatorname{sen} \alpha$ o $W=|\vec{F} \| \Delta \vec{r}|$ ?

Por lo que respecta a la concepción de energía, ningún profesor la relaciona con los cambios o transformaciones que experimentan los sistemas; todos la relacionan con el trabajo:

Les digo que energía es la capacidad para hacer trabajo. (Profesor 1)

Ciertamente, podemos ver aquí una idea cualitativa, pero resulta escasamente útil para argumentar con ella, pues no se ha introducido una concepción cualitativa de trabajo. Para constatar si realmente los profesores reconocen en dicha afirmación una idea cualitativa, les hemos preguntado si plantean en sus clases situaciones que deban ser abordadas a partir de ella. Un profesor afirma haberlo hecho en alguna ocasión; los otros cuatro indican no hacerlo nunca:

Yo defino la energía de la manera que viene en el libro de texto y paso rápidamente a otra cosa. De hecho, nunca pregunto en los exámenes qué entienden por energía, porque lo veo muy complicado. (Profesor 3) 
3. Analizar el significado cotidiano de los conceptos que se introducen en las clases permite sacar que lo diferencia del significado científico. Cuando les hemos preguntado si analizan el significado cotidiano de trabajo, uno de los profesores manifiesta no hacerlo:

En principio, no [hago referencia a este aspecto]. Igual hacemos algún ejercicio, como, por ejemplo, si hace trabajo una persona que sostiene una maleta, pero sin darle demasiada importancia. El trabajo cotidiano no lo comento. (Profesor 2)

Los otros cuatro afirman tratar las diferencias entre ambos significados, pero en todos los casos lo hacen una vez introducida la definición operativa de trabajo y para mostrar que no hay ninguna relación entre ambos:

Entrevistador ¿Haces referencia a lo que se entiende por trabajo en la vida diaria?

Profesor 1 Sí. Les pregunto, ¿qué entendéis por trabajo? Y ellos me ponen ejemplos que realmente no son trabajo, sino un esfuerzo físico.

Entrevistador ¿ ¿Y cómo lo distingues?

Profesor 1 Pues pongo el caso en que el trabajo es cero como, por ejemplo, cuando empujamos una pared.

Entrevistador $\quad$ ¿Y esto lo haces cuando ya has definido operativamente el trabajo o antes?

Profesor 1 Después. Porque ellos me dicen: se hace mucho trabajo al estudiar y yo les explico la expresión... y a partir de ahí lo deducimos todo. Porque ellos asocian trabajo a fuerza.

Profesor 5 Sí que hago referencia a lo que se entiende por trabajo en la vida diaria.

Entrevistador Pero eso, ¿̇después de disponer de la definición operativa de trabajo?

Profesor 5 Sí, claro, después de haberlo definido, les digo «como veis, en física, la idea de trabajo no tiene nada que ver con el trabajo en la vida cotidiana».

Nada habría que objetar a este planteamiento a no ser porque, de nuevo, manifiesta un aborde puramente operativo, sin aportar argumentos cualitativos, análisis que evidentemente no pueden hacerse a causa de la «arbitrariedad» de la definición cuantitativa de trabajo propuesta.

Al constatar las diferencias entre los significados diario y científico de trabajo, se pone de relieve la existencia de una ruptura, que evidentemente existe, entre ambos, pero pensamos que sería más adecuado que los alumnos notasen que los científicos partieron del significado diario para llegar a su concepción actual (Kuhn, 1983): las dificultades del concepto cotidiano para resolver los problemas ha llevado a matizaciones, puntualizaciones, limitaciones, etc., con el resultado de que actualmente se trata de concepciones esencialmente distintas. Si no fuese así, ¿por qué utilizar el mismo término para significados tan distintos?

4. Los cinco profesores coinciden en la importancia de la conexión del trabajo con la variación de energía, pero todos manifiestan tener dudas acerca de que los estudiantes la adquieran.

Entrevistador En tu opinión, ¿qué debería saber un estudiante acerca del trabajo?

Profesor 1 Lo deberían ligar a la energía. Ahí es donde adquiere significado, pero ellos no lo suelen relacionar... Les cuesta mucho. Has de hacerles muchas referencias a la energía y decirles que lo vean desde diferentes puntos de vista, es decir, ¿̨ha habido variación de altura?, ¿̨ha habido variación de velocidad?... Pero eso no lo saben ligar. 
Cuando se les ha preguntado si proponen alguna situación en la que deban calcular el trabajo realizado a partir de la variación de energía experimentada por el sistema, los cinco contestan negativamente:

Entrevistador ¿Les propones alguna situación en la que tengan que calcular el trabajo a partir, no de la expresión $W=|\vec{F}||\Delta \vec{r}| \cos a$, sino de la variación de energía experimentada por el sistema?

Profesor $5 \quad$... No, la verdad es que no se lo he pedido nunca.

Puesto que una constatación de la investigación didáctica es el poco uso que hacen los alumnos de las relaciones trabajo y energía a la hora de enfrentarse a la resolución de problemas de movimiento (Doménech y Martínez-Torregrosa, 2010), les hemos pedido razones al respecto. Todos apuntan el escaso tiempo que le destinan a este aspecto:

Creo que conceptualmente no se trabaja suficientemente eso de la energía. Los alumnos no lo asimilan y entonces van a la cinemática y dinámica. Tal vez sea porque la energía se toca con pinzas y seguramente se debería incidir más. Creo que, en general, el concepto de energía queda confuso. Creo que los profesores le dedicamos tiempo a la cinemática y la dinámica, pero huimos de la energía. (Profesor 5)

Un profesor señala las confusiones de los alumnos acerca de los conceptos involucrados:

En las relaciones trabajo y energía, ellos no saben si se trata del trabajo hecho por todas las fuerzas, por las exteriores, las conservativas, las interiores, etc. Este es un error que he comprobado que tienen. Tienen mucha confusión. Les hago resolver algunos ejercicios de las dos maneras pero no consigo... pero es que se trata de cosas muy complicadas... Igual si le dedicáramos más tiempo... (Profesor 3)

Otro apunta, además, dificultades de comprensión por parte de los profesores:

Creo que no hemos enfocado el tema correctamente, por falta de tiempo, por desconocimiento nuestro, etc. No sé si es porque nosotros no le damos la importancia que se merece o que se trata de un concepto que no saben adquirir bien. Solo llegan a entender el nombre de energía potencial y calcularla y el nombre de energía cinética y calcularla. (Profesor 1)

Lo hacen porque la cinemática y la dinámica ya las han superado, pero la energía es una cosa nueva y no lo tienen demasiado claro. Ellos no ven que a veces es mucho más fácil. Puede ser que si empezáramos por energía y después tratáramos la cinemática lo conseguiríamos. Tal vez si le dedicáramos más tiempo... Y eso sin contar que igual no lo tengan claro... No sé, al acabar el tema, como máximo llegan a la fórmula del trabajo. Creo que no enfocamos el tema de energía como se merece. Por falta de tiempo, por desconocimiento nuestro, porque no lo hacemos práctico, porque es más abstracto, etc. (Profesor 4)

A la pregunta de si los alumnos atribuirán la energía potencial de un objeto lanzado al aire al conjunto objeto-Tierra y no solo al objeto, todos indican que, a pesar de tratarlo en sus clases, la mayoría de los alumnos pensarán que la energía potencial corresponde únicamente al objeto. Al pedirles alguna razón, los cinco profesores apuntan al escaso tiempo que se le dedica:

Entrevistador ¿Qué crees que entenderán los estudiantes cuando se les indica que la energía potencial para el caso de un objeto de $1 \mathrm{~kg}$ situado a una altura de $1 \mathrm{~m}$ de la superficie terrestre es de $10 \mathrm{~J}$ ?

Profesor 3 Solamente verán un número.

Entrevistador ¿ ¿No dirán nada más? 
Profesor 3 Un alumno que piense dirá que es la energía del cuerpo. Ahora, ¿qué es la energía para ellos?, no lo sé.

Entrevistador ¿Pensarán que la energía pertenece tanto al objeto como a la Tierra?

Profesor 3 Lo deberían de pensar, ¿no? (...) pero no... dirán que es la energía del cuerpo en este punto y ya está.

Entrevistador ¿iY a qué puede ser debido?

Profesor 3 Puede ser que no se insista demasiado.

Por lo que se refiere a la imposibilidad de determinar la energía de un sistema, los cinco afirman tratar este aspecto, aun cuando de nuevo indican el poco tiempo que le destinan como una razón del desconocimiento por parte de los alumnos:

Entrevistador ¿Abordas en clase el hecho de que no podemos determinar, de una manera absoluta, la energía de un sistema, sino solo sus variaciones?

Profesor 1 Sí que lo trato, pero creo que no llegan a captarlo. Creo que aquí tienen mucha responsabilidad los textos, porque llegan a pedir a los alumnos que calculen la energía cinética de un punto y les hacen pensar en la fórmula sin el incremento.

Entrevistador ¿Y le dedicas el tiempo suficiente?

Profesor 1 Creo que no. Es que este tema no se trata con la suficiente profundidad.

Entrevistador ¿Abordas en clase el hecho de que no podemos determinar, de una manera absoluta, la energía de un sistema, sino solo sus variaciones?

Profesor 2 Lo comento. Si hemos dicho que el movimiento es relativo, que un cuerpo puede tener cualquier velocidad, dependiendo del sistema de referencia, entonces ¿qué ocurre?, que cada vez tiene una energía cinética. Quiero decir, que el valor real no lo podemos saber nunca.

Entrevistador ¿A esto le dedicas el tiempo suficiente?

Profesor $2 \quad$ No, simplemente lo comento una vez, al principio.

Entrevistador ¿Crees que los estudiantes se apropiarán de esta idea?

Profesor 2 No, creo que ellos pensarán en un valor absoluto. Pienso que deberíamos insistir más en este aspecto.

Un profesor afirmó desconocer esta imposibilidad:

Nunca he trabajado de esta forma; siempre lo he hecho, erróneamente por lo que veo, determinando valores absolutos de la energía cinética y de la potencial de un objeto en un punto determinado. (Profesor 4)

En relación con el calor, los profesores no creen que los estudiantes perciban su relación con la variación de energía. Varios apuntan a la complejidad del concepto:

Entrevistador ¿Qué crees que debería saber un estudiante acerca del calor?

Profesor 5 Que no es un fluido. Que sepan que es una forma de transferir energía cuando los cuerpos están a diferente temperatura. Pero, claro, el concepto de calor es complicado. Incluso el mismo concepto de temperatura también lo es.

Otro se refiere, además, al carácter marcadamente operativista de la enseñanza y a un tratamiento superficial: 
Entrevistador ¿Qué crees que debería saber un estudiante acerca del calor?

Profesor 4 Debería saber que se trata de otra forma de transmitir energía de un cuerpo a otro, que se produce a causa de una diferencia de temperaturas... que pasa de un cuerpo caliente a otro frío... Energía, trabajo y calor son conceptos interrelacionados que se prestan a confusiones, tanto por parte del alumnado como del profesorado... El concepto de calor tal vez los alumnos lo confundan con el de temperatura, y entonces deberían distinguirlos... pero nosotros solo damos la fórmula en que aparece la relación con la temperatura y la masa... Realmente el calor se introduce en un tema distinto del de trabajo y energía.

Entrevistador ¿Cuando estudias los fenómenos caloríficos, retomas los conceptos de trabajo y energía?

Profesor $4 \quad$ No, no los retomo, no los relaciono... hablo un poco de la energía, pero me voy al concepto de calor y dejo de lado la energía.

Un profesor afirma no introducir el concepto de calor:

Yo nunca hablo de calor... siempre hablo de energía térmica... Los libros siempre hablan un poco... pero últimamente muy poco... El calor lo hemos desterrado. (Profesor 3)

La expresión $\mathrm{W}+\mathrm{Q}=\Delta \mathrm{E}$ no solo resume la integración de dos campos del conocimiento (mecánica y termodinámica), sino que conduce, para el caso de un sistema aislado, al principio de conservación de la energía. Se trata de una relación importante y hemos preguntado si la utilizan en sus clases. Algunos afirman utilizarla en Química, pero ninguno lo hace en la materia de Física, ni tan siquiera en el tema en el que introduce el principio de conservación de la energía. En los argumentos, los profesores muestran confusiones acerca del calor y de la energía interna:

Entrevistador ¿Utilizas la expresión $\mathrm{W}+\mathrm{Q}=\Delta \mathrm{E}$ ?

Profesor $1 \quad$ No. A veces los alumnos sí que utilizan $\mathrm{Q}=\Delta \mathrm{E}$ y $\mathrm{W}=\Delta \mathrm{E}$, pero así completa no.

Entrevistador $\quad$ ¿Y no planteas problemas como, por ejemplo, soltar un objeto desde una altura y pedirles que determinen el aumento de temperatura que experimenta al chocar con el suelo?

Profesor 1 Sí, pero lo hacen por separado, primero utilizan $\mathrm{W}=\Delta \mathrm{E} y$, si el trabajo se convierte en calor, dicen ellos, después hacen $\mathrm{Q}=\Delta \mathrm{E}$. Ellos calculan por un lado el calor y después piensan: ¿qué, o gracias a qué, se ha podido hacer esto? Pues gracias al hecho de que ha caído, por ejemplo, un martillo. Por tanto, ha experimentado una variación de energía, se ha convertido en... pero nunca se presenta la situación de realizarse un trabajo y un calor al mismo tiempo.

Los cinco profesores afirman introducir el principio de conservación de la energía. Puesto que en todos los casos lo introducen al estudiar movimientos en presencia de la fricción, están poniendo de relieve la confusión entre el principio de conservación de la energía (que tiene en cuenta todas las diferentes formas de energía y es de validez general) y la expresión a la que conduce el teorema de las fuerzas vivas para el caso en que todas las fuerzas actuantes sean conservativas (expresión que no tiene, por tanto, validez general): 
Entrevistador ¿Cuándo introduces el principio de conservación de la energía?

Profesor 2 Una vez introducida la energía cinética, la potencial y las fuerzas conservativas, demuestro que, cuando todas las fuerzas son conservativas, la suma de la energía cinética y la potencial es siempre constante.

Entrevistador $\quad$ ¿Y a esto le llamas principio de conservación de la energía?

Profesor 2 Sí, este es el principio de conservación de la energía en mecánica.

Algunos razonan como si, al hacer uso de dicho principio, estuviese en nuestras manos no prestar atención a algunas formas de la energía:

Entrevistador ¿En qué momento introduces el principio de conservación de la energía?

Profesor 4 En mecánica.

Entrevistador ¿Antes de estudiar el calor?

Profesor 4 Sí.

Entrevistador ¿Y queda como general?

Profesor 4 Sí, es que el calor no lo consideramos... Ahora creo que lo mezclo con la energía mecánica... no sé, ahora veo algunos inconvenientes.

Les hemos preguntado si abordan en sus clases la aparente contradicción que existe entre el principio de conservación de la energía del que se habla en física y el agotamiento de la energía del que hablan los medios de comunicación. Ningún profesor plantea dicha contradicción a sus alumnos:

No lo trato. Seguramente debería hacerlo pero no lo he hecho nunca. Seguramente no he entrado nunca en este aspecto porque las dificultades de los estudiantes no llegan a nosotros... A lo mejor es por falta de intercambio, de comunicación... A veces les sueltas el principio de conservación de la energía y ellos lo cogen y punto. Igual después lo reflexionan y ven dificultades, pero no te llega... Pero veo que es un problema. (Profesor 4)

Hemos de señalar que dos profesores reconocen que se trata de una paradoja que nunca se habían planteado. Uno afirma tener dificultades para resolverla:

Esto es difícil incluso para los propios profesores. Yo, si no surge el tema no lo abordo... (Profesor 1)

El otro razona como si se tratase de un problema de lenguaje, siendo así que se trata de dos ideas que quedan recogidas en dos principios distintos de la termodinámica:

Esta contradicción no me la han planteado nunca. Si me la planteasen les diría que una cosa es el lenguaje en el aula y otra el de la calle, y que a veces no coincide. (Profesor 3)

Hemos intentado averiguar en qué medida los profesores salen al paso de posibles preconcepciones de los estudiantes. Concretamente, les hemos planteado si cuestionan el carácter sustancial que en la vida diaria se le atribuye a la energía, y si distinguen entre energía interna y calor. Algunos afirman tratar estos aspectos en sus clases pero tienen dudas respecto a que sus alumnos superen las posibles preconcepciones, pues no lo hacen con el suficiente detenimiento: 
Entrevistador ¿La continua utilización de expresiones como, por ejemplo, «para que un coche funcione hace falta energía», "cuando una bola en movimiento choca con otra en reposo le transfiere energía», etc., puede hacer creer a los alumnos que la energía es una especie de fluido contenido en los cuerpos? ¿Abordas este aspecto en tus clases y haces ver a los alumnos que se trata, simplemente, de una manera de hablar?

Profesor 5 Sí que lo digo, sí que lo comento.

Entrevistador ¿Crees que los alumnos lo asumirán?

Yo lo trato, pero no estoy seguro de que terminen con esta interpretación, porque solo lo trato un poco.

Un profesor indica no haberse dado cuenta de este aspecto:

Entrevistador

Profesor 4
¿Abordas el posible carácter sustancial que los alumnos pueden atribuir a la energía?

No, creo que no. De hecho, si... ni yo me había dado cuenta de esta manera de hablar.

\section{CONCLUSIONES}

Los resultados obtenidos con la cuestión abierta y las entrevistas confirman la hipótesis según la cual los profesores no tratan con la profundidad suficiente ni tan siquiera los aspectos conceptuales relacionados con la energía, siendo así que se trata de aquella parcela a la que suelen limitarse.

Como cabía esperar, existen diferencias entre los profesores en activo y los que están en formación respecto de lo que supone una comprensión adecuada de la energía, pero las diferencias deberían ser más acusadas. De una manera general, podemos decir que para los profesores en formación la comprensión de la energía pasa básicamente por su conservación. Estos pobres resultados no solo cuestionan el tipo de enseñanza en secundaria, sino también la universitaria.

Aun cuando los profesores afirman tratar en sus clases un número considerable de los aspectos que consideramos pertinentes, como indican en las entrevistas, el tratamiento dado es muy superficial.

En particular, podemos referirnos a que los conceptos que se elaboran no responden al intento de resolver problemas importantes, lo cual no facilita su comprensión. Coherentemente, los conceptos se introducen vía definiciones cuantitativas, sin aportar ninguna razón al respecto. Esta estrategia hace que los alumnos no perciban la racionalidad del trabajo científico: parece como si las definiciones operativas «cayesen del cielo» y no que fuesen el fruto y la concreción de intuiciones cualitativas.

Aceptando que los significados científico y cotidiano del trabajo son muy distintos, la manera como se aborda este concepto en las clases hace de la ciencia un campo alejado de la realidad en la que estamos inmersos; lejos de ser así, la ciencia intenta resolver problemas cotidianos. Además, en este caso concreto, ligar el concepto de trabajo con la variación de energía de un sistema justifica la definición operativa $W=|\vec{F}||\Delta \vec{r}| \cos \alpha$ (válida para el caso de un objeto que experimenta cambios de posición cuando se ejerce una fuerza constante sobre él). Al hacer esto mejoramos la comprensión del concepto trabajo (y la de energía) y proporcionamos una imagen más ajustada de lo que supone el avance científico.

Los profesores no le dedican, cuando lo hacen, el suficiente tiempo a poner en cuestión las posibles concepciones alternativas de los alumnos. Es más, hemos de reconocer que la estrategia de enseñanza utilizada favorecerá, al menos en algunas ocasiones, la consolidación de dichas ideas. Así, por ejemplo, al no poner énfasis en la energía interna, los alumnos atribuyen al calor un doble significado: el calor 
es, además de una manera de cambiar la energía de los sistemas, una medida de lo caliente que se encuentra un objeto. Y no se trata, como afirma un profesor, de no introducir el concepto de calor y razonar únicamente en términos de energía interna. Se trata de dos conceptos importantes y útiles, y no hemos de esconder las diferencias entre ambos.

Hemos de resaltar que los profesores suelen mostrarse pesimistas respecto a las posibilidades de que los estudiantes alcancen una comprensión significativa de todo aquello relacionado con la energía, pues eso constituye el primer paso para que efectivamente dicha profecía se cumpla.

El hecho más evidente de la superficialidad de la enseñanza de la energía lo constituye la reiteración al poco tiempo que dedican a tratar aspectos que consideran importantes y respecto de los cuales tienen dudas del aprendizaje conseguido por los alumnos. Si están convencidos de la conveniencia de una enseñanza en profundidad, ¿por qué no detenerse y facilitar el aprendizaje?, ¿por qué no abordar con detenimiento las relaciones entre los conceptos?

Por último, nos referiremos las dificultades que los propios profesores reconocen acerca de algunos de los aspectos relacionados con la energía (imposibilidad de determinar valores absolutos de la energía, necesidad de explicitar el sistema físico al que se presta atención, qué es la energía, diferencias entre el teorema de las fuerzas vivas y la expresión $\mathrm{W}+\mathrm{Q}=\Delta \mathrm{E}$, etc.). Por supuesto, estas carencias no facilitan el aprendizaje. Uno de los acuerdos entre los investigadores es que la buena enseñanza exige un dominio de la materia por parte de los profesores.

Dejamos para otro trabajo mostrar que el aprendizaje alcanzado por los estudiantes mejora considerablemente en una enseñanza que contempla todos los aspectos que se han apuntado.

\section{REFERENCIAS BIBLIOGRÁFICAS}

Ausubel, D. P., NovaK, J. D. y Hanessian, H. (1983). Psicología educativa: un punto de vista cognoscitivo. México: Trillas.

ARONS, A. B. (1997). Teaching in introductory physics. USA: J. Wiley.

BeYnOn, J. (1990). Some myths surrounding energy. Physics Education, 25, pp. 314-316.

BROWN, H. I. (1988). La nueva filosofía de la ciencia. Madrid: Tecnos.

CAMPELO, J. R. (2003). Un modelo didáctico para enseñanza aprendizaje de la física. Revista Brasileira de Ensino de Física, 25(1), pp. 86-104.

Chalmers, A. (1984). ¿Qué es esa cosa llamada ciencia? Madrid: Siglo XXI.

Chalmers, A. (1992). La ciencia y cómo se elabora. Madrid: Siglo XXI.

Chi, M., Feltovich, P. J. y Glaser, R. (1981). Categoritation and representation of physics problems experts and novices. Cognitive Science, 5, pp. 121-151.

CHI, M., Glaser, R. y FARR, M. (1982). Expertise in problem solving. En Stenberg (ed.). Advances in the psychology of human intelligence (1). Hillsdale, N.J.: Erlbaum.

Chisholm, D. (1992). Some energetic thoughts. Physics Education, 27, pp. 215-220.

De Oliveira, E. T. Caballero, C. y Moreira, M. A. (2006). Possíveis indicadores de invariantes operatórios apresentados por estudiantes em conceitos da termodinâmica. Revista Brasileira de Ensino de Física, 28(4), pp. 463-471.

DOMÉNECH, J. L. (2000). L'ensenyament de l'energia en l'educació secundària. Anàlisi de les dificultats i una proposta de millora. Tesi doctoral. Universitat de València.

Doménech, J. L., Gil-PÉreZ, D., Gras, A., Guisasola, J., MartíneZ-Torregrosa, J. y Salinas, J. (2001). La enseñanza de la energía en la educación secundaria. Un análisis crítico. Revista de Enseñanza de la Física, 14(1), pp. 45-60. 
Doménech, J. L., Gil-Pérez, D., Gras, A., Guisasola, J., MartíneZ-Torregrosa, J., Salinas, J., Trumper, R., VAldés, P. y Vilches, A. (2007). Teaching of Energy Issues: A Debate Proposal for a Global Reorientation. Science \& Education, 16, pp. 43-64.

DOMÉNECH, J. L. y MARTÍNEZ-TORREGROSA, J. (2010). ¿Los estudiantes de secundaria terminan sus estudios con una comprensión adecuada de los conceptos de trabajo, calor y de su relación con la energía? Revista Brasileira de Ensino de Física, vol. 32, núm. 1, 1308, 10 pp.

DRIVER, R. (1988). Un enfoque constructivista para el desarrollo del currículo de ciencias. Enseñanza de las Ciencias, 6(2), pp. 171-176.

DUIT, R. (1986). In search of an energy concept. En Driver, R. y Millar, R. (eds.). Energy matters. University of Leeds.

DUSCHL, R. A. (1997). Renovar la enseñanza de las ciencias. Madrid: Narcea.

Gil, D., Moreira, M. A., DiAS, C. y GARret, R. (1992). Proyecto IBERCIMA. Recomendaciones para el diseño de los currículos de ciencias. Revista Brasileira de Ensino de Física, 14(4), pp. 257259.

HARMAn, P. M. (1990). Energía, fuerza y materia. Madrid: Alianza.

KuHN, T. S. (1983). La tensión esencial. México: FCE.

LARKin J. H., MCDermotT, J., Simon, D. P. y SimON, H. A. (1980). Expert and novice perfomance in solving physics problems. Science Education, 208, pp. 1335-1342.

LINN, M. C. (1987). Establishing a research base for science education: challenges, trends and recommendations. Journal of Research in Science Teaching, 24, pp. 191-216.

MallinCKRodT, A. J. y LefF, H. S. (1992). All about work. American Journal Physics, 60 (4), pp. 356-365.

NovaK, J. D. (1998). Conocimiento y aprendizaje. Madrid: Alianza Editorial.

OGBORN, J. (1986). Energy and fuel -the meaning of «the go of things». En Driver R. y Millar, R. (eds.). Energy matters. University of Leeds.

Osborne, R. y WiTtrock, M. (1983). Learning science: a generative process. Science Education, 67, pp. 5-14.

Osborne, R. y WitTrock, M. (1985). The generative learning model and implications for science education. Studies in Science Education, 12, pp. 59-87.

PRIDEAUX, N. (1995). Different approaches to the teaching of the energy concept. SSR, 77, pp. 49-57.

SOLBES, J. y TARín, F. (1998). Algunas dificultades en torno a la conservación de la energía. Enseñanza de las Ciencias, 16 (3), pp. 387-397.

SOlBES, J. y TARÍn, F. (2004). La conservación de la energía: un principio de toda la física. Una propuesta y unos resultados. Enseñanza de las Ciencias, 22(2), pp. 185-294.

TRUMPER, R. y GORSKY, P. (1993). Learning About Energy: The Influence of Alternative Frameworks. Cognitive Levels, and Closed-Mindedness. Journal of Research in Science Teaching, 30(7), pp. 637648.

VARELA, P. y PÉREZ, M. C. (2006). Una propuesta para desarrollar en el alumno de secundaria una visión unificada de la física a partir de la energía. Revista Eureka sobre enseñanza y divulgación de las ciencias, vol. 3(6), pp. 237-250.

Vosniadou, S., Ionnides, C. Dimitrakopoulou, A. y Papademetriou, E. (2001). Designing learning enviroments to promote conceptual change in science. Learning and Instruction, 11, pp. 381-419.

WELTI, R. (2005). Obstáculos conceptuales en el aprendizaje de la energía de las ondas. Revista Brasileira de Ensino de Física, 27(3), pp. 487-490. 


\section{APÉNDICE}

\section{Aspectos que tratar en las entrevistas}

1. Sabemos que, por ejemplo, los conceptos de velocidad y aceleración se introducen en clase con el propósito de describir los movimientos y que el concepto de fuerza se introduce con el objetivo de explicar los cambios de movimiento experimentados por los objetos, pero, ¿qué pretendemos explicar con el concepto de energía? ¿Qué les dices a tus alumnos?

2. Si quisiéramos que un alumno dispusiese de una concepción adecuada de la energía, ¿qué crees que debería saber?

3. Seguramente has oído hablar de las dificultades a la hora de definir la energía. ¿Propones alguna definición de este concepto? ¿Cuál? Si la defines como la capacidad para realizar un trabajo, ¿les propones alguna situación que tenga que ser resuelta a partir de la idea de que la energía es la capacidad para realizar trabajo?

4. ¿Abordas en clase el hecho de que no podemos determinar, de una manera absoluta, la energía de un sistema, sino solo sus variaciones? ¿Le dedicas el tiempo suficiente?

5. ¿Qué crees que entenderán los estudiantes cuando se les indica que la energía potencial para el caso de un objeto de $1 \mathrm{~kg}$ situado a una altura de $1 \mathrm{~m}$ de la superficie terrestre es de $10 \mathrm{~J}$ ?

6. Haces referencia al poco sentido que tiene hablar de la energía potencial de un único objeto, y que realmente dicha energía corresponde al conjunto objeto y Tierra?

7. La continua utilización de expresiones como, por ejemplo, «para que un coche funcione le hemos de proporcionar energía», "cuando una bola en movimiento choca con otra en reposo le transfiere energía», etc., puede hacer creer a los alumnos que la energía es una especie de fluido contenido en los objetos. ¿Abordas este aspecto en las clases? ¿Haces a tus alumnos conscientes de que se trata solamente de una manera de hablar?

8. ¿Cuándo introduces el principio de conservación de la energía? El principio de conservación de la energía es un aspecto básico de la física; no obstante, el hecho de que continuamente estemos utilizando expresiones que parecen contradecirlo («la energía del planeta se está agotando», "para hacer funcionar los motores hemos de consumir energía») podría llevar a los alumnos a no aceptar plenamente su validez. ¿Tratas esta aparente contradicción en tus clases?

9. El concepto de trabajo suele introducirse en el tema de energía. ¿Introduces alguna idea cualitativa sobre trabajo? ¿Cómo introduces el trabajo? ¿Haces referencia a lo que se entiende por trabajo en la vida diaria? ¿De qué manera lo haces?

10. Habrás notado que los alumnos no suelen utilizar las relaciones trabajo-variación de energía para enfrentarse a los problemas de movimiento, ¿a qué atribuyes estas reticencias? ¿Qué haces para que las usen?

11. Con relación al calor, ¿qué crees que debería saber un estudiante acerca del calor?

12. ¿Introduces la expresión $W+Q=\Delta E$ ? ¿Planteas problemas como, por ejemplo, soltar un objeto desde una altura y pedir que determinen el aumento de temperatura que experimenta al chocar con el suelo? 


\title{
SUPERFICIAL TEACHING OF THE SCIENCE CONCEPT ENERGY: A POSSIBLE CAUSE OF REDUCED STUDENT LEARNING AT THE COMPLETION OF HIGH SCHOOL
}

\author{
Josep Lluís Doménech, Rubén Limiñana, Asunción Menargues \\ Departamento de Didáctica General y Didácticas Específicas \\ Universidad de Alicante \\ jl.domenech@ua.es, ruben.Im@gmail.com, a.menargues@ua.es
}

Students' low understanding of the science concept energy, at the completion of high school, lead this research team to investigate conceptual frameworks and teaching methods related to energy. It is generally understood that alternative teaching methods can result in better understanding of scientific concepts. However, this paper focuses solely on how the concept of energy is taught in traditional science education.

In science education, superficial teaching commonly occurs in courses covering many aspects or concepts of a given topic. Researchers are in agreement that only a limited number of concepts need to be taught in more depth, rather than superficially covering an even higher number of concepts. Given the inherent complexity of scientific concepts, it is more convenient for teachers to identify students' interest and the relevancy of specific problems. From this the required concepts, their usefulness to solve general problems, and the social and technological implications can be assessed.

This paper presents one of the main causes of low student learning of the energy concept in high school students (17-18 years old). Traditional teaching often does not take into account the important aspects (mentioned above) related to the concept of energy. Therefore, the concept is discussed superficially, preventing students developing adequate knowledge of the concept. Effective teaching of the energy concept needs to consider that:

a) Energy is a useful concept, especially in studying transformations occurring in daily life.

b) It is necessary to clarify the physical system that is being studied.

c) Only determined energy variations can be used, not absolute energy values.

d) Work and heat are two ways to modify the energy of a given system.

e) Internal energy is a different concept than heat.

f) In isolated systems, the energy remains constant.

g) As a consequence of interactions with other systems, the energy of a given system is "degraded".

h) Students may have alternative conceptual understandings of the energy concept.

To test the hypothesis (i.e. the energy concept is not usually discussed in depth), three groups of Physics and Chemistry teachers were selected for the research project. One group consisted of 31 teachers in a formative stage, the second was composed of 28 active teachers (currently teaching Physics) and the third group consisted of 5 experienced teachers (with more than five years experience, teaching Physics). Teachers from the two first groups were asked to make a list of the most important aspects in teaching the concept of energy (this was the previous question). To ascertain the aspects related to energy that are taught to students, five teachers were interviewed. The interview duration was a maximum of 45 minutes, with teachers asked a total of 12 questions. Questions were based on teachers' answers to the initial question (i.e. the most important aspects in teaching the concept of energy) and were modified after consultation with two experts.

The results indicate that high school teachers of Physics do not include all of the relevant aspects related to energy in their lessons. Moreover, in the cases where all the relevant aspects are considered, the total amount of time allocated is relatively low and therefore the concepts are only superficially discussed. The aspects discussed do not attempt to solve important problems, preventing students from obtaining a comprehensive conceptual understanding. Teachers limit the lessons to the study of the operative definitions of concepts related to energy and the Conservation of Energy Principle. Additionally, certain teachers may have difficulties in understanding some concepts, which prevents them from discussing the concept more in-depth with students. Finally, it is worthwhile to note that teachers generally think they invest a low amount of time in discussing certain aspects related to energy. Despite this, they consider these concepts to be important in high school science education. 